\title{
Introduction to the Special Section on Internet and Network Economics
}

\author{
Xiaotie Deng • Fan Chung Graham
}

Published online: 9 September 2010

(C) Springer Science+Business Media, LLC 2010

Keywords Algorithms · Computation · Economics · Finance

In this special section of Algorithmica, we have included several articles based on presentations made, during December 12-14, 2007, at the third Workshop on Internet and Network Economics (WINE 2007) in San Diego, California.

The workshop provided a forum for researchers as well as practitioners to exchange innovative ideas and to learn about each other's efforts and results. It reflects an emerging trend in merging theories, methodologies, and applications in computer science, economics, mathematics, management sciences to manage increasing problems motivated by Internet activities.

The special section chose to focus on the market problems that have attracted growing attention in recent years. Four articles covering two recent active topics have been included, covering the prediction market and the sponsored search market. All selected articles have been significantly improved from the workshop presentations, taking into consideration helpful feedback from the conference presentations and reviewers' reports.

Chen, et al., made a thorough study of user strategic behavior on the prediction market with market scoring rule market makers. While truthful behavior under myopic traders was known, they studied in this work the non-myopic strategies of traders and developed information structures that lead to truthful behavior, for both the finite time horizon model and the infinite time horizon model.

\footnotetext{
X. Deng $(\varangle)$

Department of Computer Science, City University of Hong Kong, Hong Kong SAR, P.R. China e-mail: deng@cs.cityu.edu.hk

F.C. Graham

Department of Mathematics, University of California, San Diego, La Jolla, CA, USA

e-mail: fan@ucsd.edu 
Three other articles are related to the active research area in sponsored search. $\mathrm{Bu}$, et al., studied the robustness of the forward looking behavior of bidders in GSP, the generalized second price protocol, a dominating protocol that has been actively analyzed in the field. Langford, et al., developed an exploration algorithm that aimed to learn click-through rates of advertisers, and at the same time, to minimize the incentive to change their current bids. Finally, Muthukrishnan, et al., formulated three stochastic models to study the stochastic evaluation problem and the stochastic budget optimization problem.

The participants at the third Workshop on Internet and Network Economics presented research results in a much wider spectrum. Our choices in this special issue have been from those most closely related to the Internet market design problem, one of the major driving forces in the flourishing and dynamic field.

We would like to thank the reviewers for the thorough feedback they provided for finalizing the articles in this special section. 